\title{
Supporting information for \\ Coupling Emitters and Silver Nanowires to Achieve Long-Range Plasmon-Mediated Fluorescence Energy Transfer
}

\author{
Juan de Torres ${ }^{1}$, Patrick Ferrand ${ }^{1}$, Gérard Colas des Francs ${ }^{2}$, and Jérôme Wenger*1 \\ ${ }^{1}$ CNRS, Aix-Marseille Université, Centrale Marseille, Institut Fresnel, UMR 7249, 13013 \\ Marseille, France \\ ${ }^{2}$ Université Bourgogne Franche-Comté, CNRS, Laboratoire Interdisciplinaire Carnot de \\ Bourgogne (ICB), UMR 6303, 21078 Dijon, France
}

E-mail: jerome.wenger@fresnel.fr

This document contains the following supporting information:

1. Absorption and emission spectra of the fluorescent nanoparticles

2. Influence of the nanowire on the nanoparticle fluorescence spectrum, detection fixed on the nanoparticle

3. Influence of the nanowire on the nanoparticle fluorescence spectrum, excitation fixed on the nanoparticle

4. Experimental setup

5. Relative contributions of the radiative, non-radiative and plasmon-mediated decay rates 


\section{Absorption and emission spectra of the fluorescent nanoparticles}
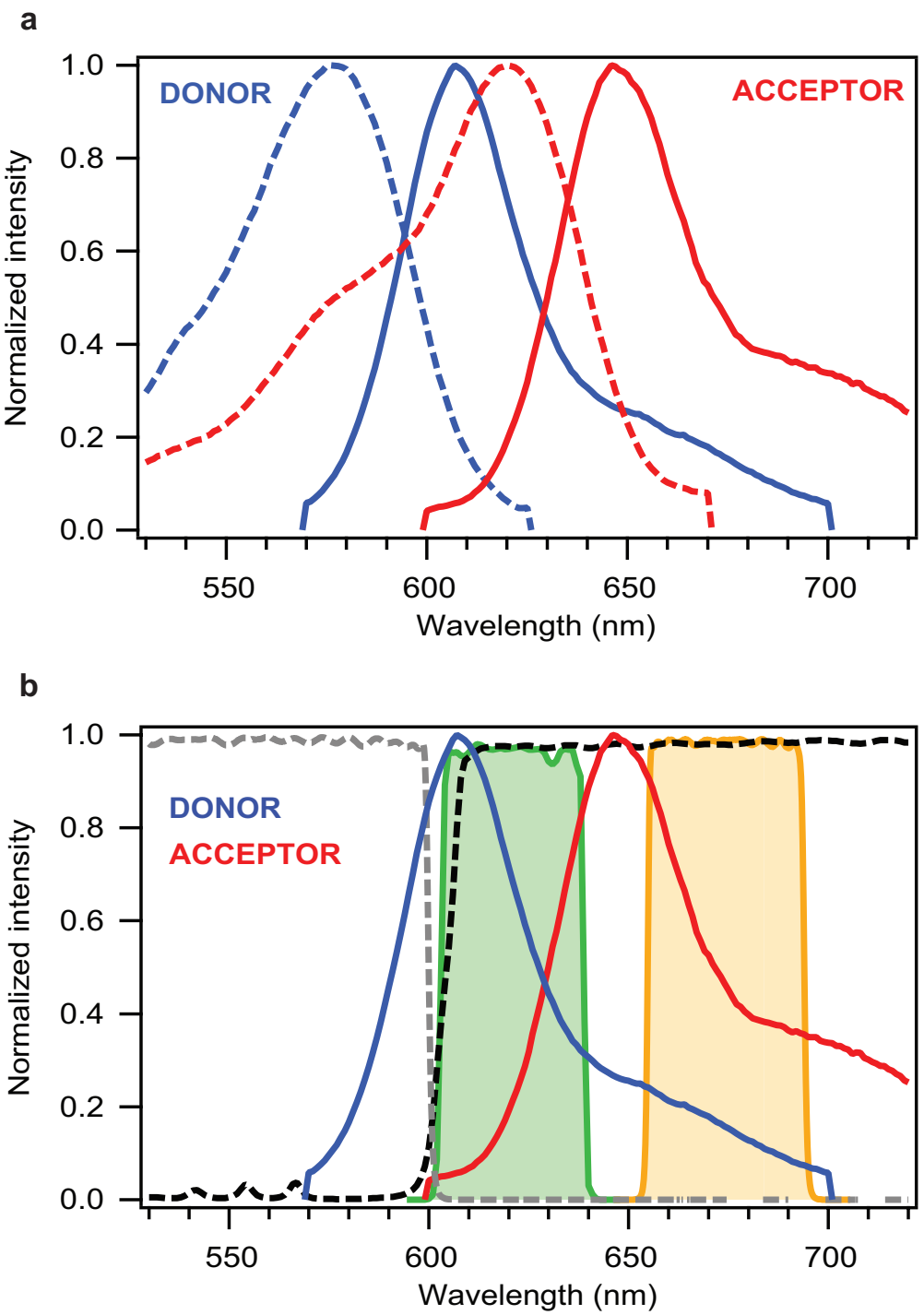

Figure S1: (a) Absorption (dashed line) and emission (solid line) spectra of donor (blue) and acceptor (red) nanoparticles. The laser excitation is set at $550 \mathrm{~nm}$ wavelength. (b) Emission spectra of the nanoparticles together with the dichroic filter (black dashed line) used to separate the donor and acceptor fluorescence from the reflected laser light, the detection bandpass filters for donor channel (green) and acceptor channel (orange) and the excitation filter (gray). 


\section{Influence of the nanowire on the nanoparticle fluorescence spec- trum, detection fixed on the nanoparticle}
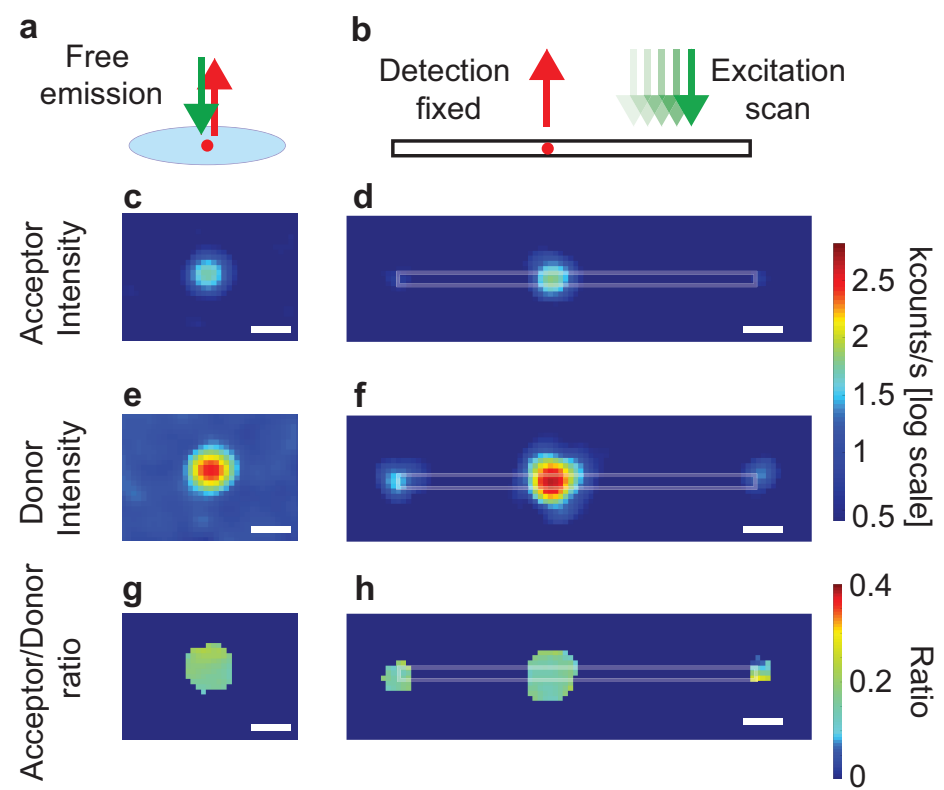

Figure S2: (a,b) Experimental schemes for (a) the free fluorescence emission with excitation and detection fixed on the donor nanoparticle immobilized on a glass coverslip and for (b) the plasmonmediated fluorescence emission with detection fixed on the donor nanoparticle in contact with the nanowire while the excitation laser is scanned all over the structure. The fluorescence images are recorded in two detection spectral channels corresponding to the acceptor emission (c,d) and the donor emission (e,f). The emission of the donor nanoparticle is collected mainly in the donor detection channel but still a part of the spectrum overlaps the detection band of the acceptor channel (crosstalk), as displayed in Fig. S1b. The ratios $(\mathrm{g}, \mathrm{h})$ of acceptor to donor intensities are computed using the corresponding images (c,e) and (d,f) to demonstrate that, at least in the detection bands limited by our filter set, the modification of the emission spectrum of the nanoparticle is negligible when the nanoparticle is excited remotely via surface plasmons and also when the nanoparticle is in contact with the nanowire and it is excited directly. Scale bars indicate $1 \mu \mathrm{m}$. 


\section{Influence of the nanowire on the nanoparticle fluorescence spec- trum, excitation fixed on the nanoparticle}

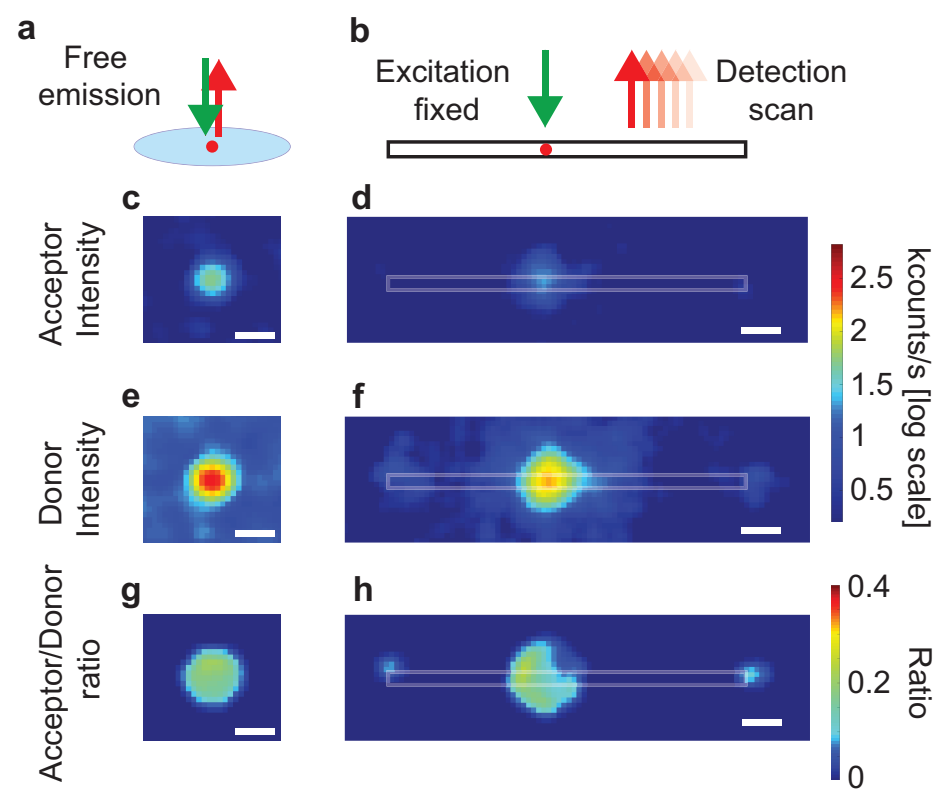

Figure S3: (a,b) Experimental schemes for (a) the free fluorescence emission with excitation and detection fixed on the donor nanoparticle immobilized on a glass coverslip and for (b) the plasmonmediated fluorescence emission with laser excitation fixed on the donor nanoparticle in contact with the nanowire while the detection is scanned all over the structure. The fluorescence images are recorded in two detection spectral channels corresponding to the acceptor emission (c,d) and the donor emission $(\mathrm{e}, \mathrm{f})$. The emission of the donor nanoparticle is collected mainly in the donor detection channel but still a part of the spectrum overlaps the detection band of the acceptor channel (crosstalk), as displayed in Fig. S1b. The ratios $(\mathrm{g}, \mathrm{h})$ of acceptor to donor intensities are computed using the corresponding images $(\mathrm{c}, \mathrm{e})$ and $(\mathrm{d}, \mathrm{f})$ to demonstrate that, at least in the detection bands limited by our filter set, the modification of the emission spectrum of the nanoparticle is negligible when the fluorescence is coupled to the metal via surface plasmons and detected at the ends of the nanowire. Scale bars indicate $1 \mu \mathrm{m}$. 


\section{Experimental setup}

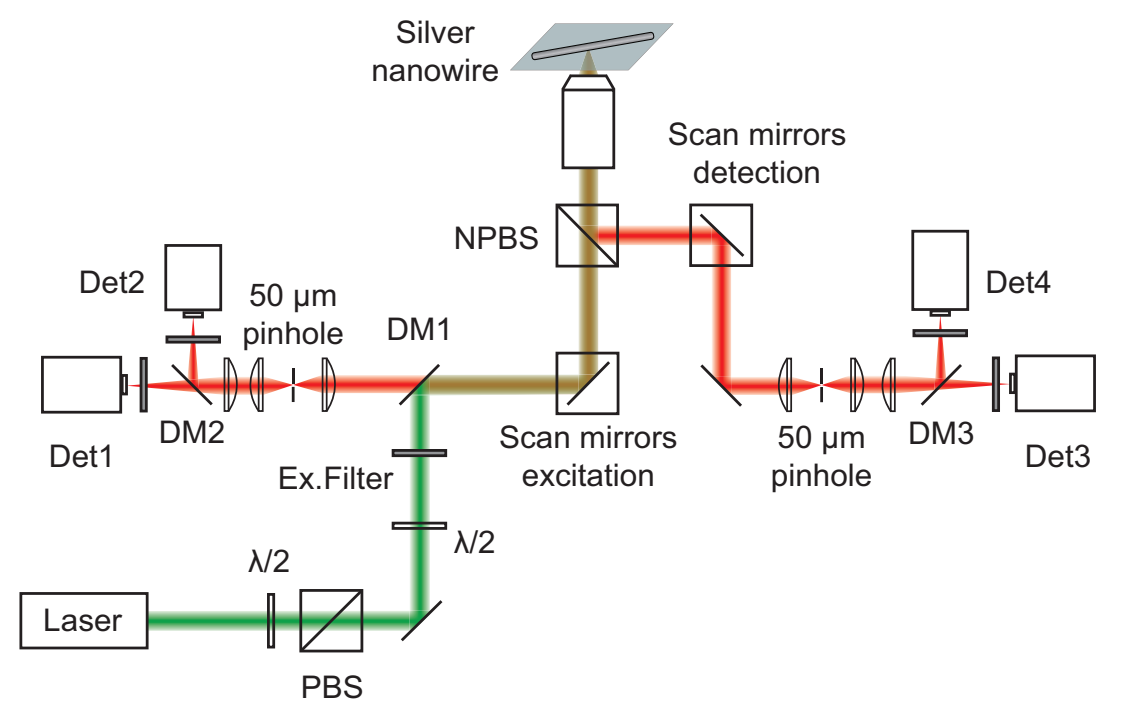

Figure S4: The excitation source is a pulsed laser at $40 \mathrm{MHz}$ repetition rate and $550 \mathrm{~nm}$ wavelength. The laser excitation is filtered by a set of two bandpass filters (Chroma ET525/70 M and Semrock FF01-550/88). The dichroic mirror DM1 (Chroma ZT594RDC) reflects the excitation wavelength and transmits the detected fluorescence. The excitation is scanned on the sample by means of a set of galvano mirrors. The light is focused on the sample with a $63 \mathrm{x} 1.2 \mathrm{NA}$ water-immersion objective and the fluorescence captured is split in two using a Non Polarizing Beam Splitter (NPBS). By means of another set of galvano mirrors the detection can be scanned independently of the excitation. Spatial filtering with a $50 \mu \mathrm{m}$ pinhole is performed. Dichroic mirrors DM2 and DM3 separate the donor and acceptor fluorescence. Finally, the detection is performed by two avalanche photodiodes with $620 \pm 20$ nm (Chroma ET605/70 M and ET632/60M) and $670 \pm 20 \mathrm{~nm}$ (Semrock FF01-676/37) fluorescence bandpass filters for the donor and acceptor channels, respectively. 


\section{Relative contributions of the radiative, non-radiative and plasmon- mediated decay rates}

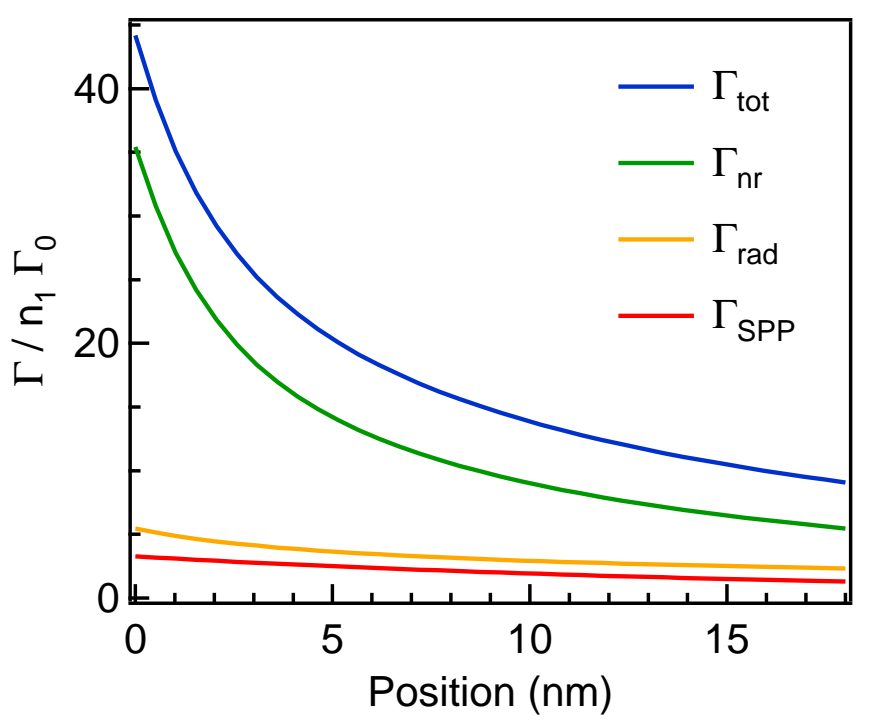

Figure S5: Total decay rate $\Gamma_{t o t}$, non-radiative (quenching) decay rate $\Gamma_{n r}$, radiative decay rate $\Gamma_{\text {rad }}$ and decay rate into the nanowire surface plasmons $\Gamma_{S P P}$ as a function of the emitter's lateral position along the line in Fig. 3a. 